\title{
Thermal analysis and design of a volumetric solar absorber depending on the porosity
}

\author{
M.I. Roldán ${ }^{\mathrm{a}, *}$, O. Smirnova ${ }^{\mathrm{b}}$, T. Fend ${ }^{\mathrm{b}}$, J.L. Casas $^{\text {c }}$, E. Zarza ${ }^{\mathrm{d}}$ \\ ${ }^{a}$ Convenio UAL-Plataforma Solar de Almería, Ctra. de Senés, km. 4.5, E04200 Tabernas, Almería, Spain \\ ${ }^{\mathrm{b}}$ Institute of Technical Thermodynamics, German Aerospace Centre (DLR), Linder Höhe, D51147 Köln, Germany \\ ${ }^{\mathrm{C}}$ Universidad de Almería, Ctra. Sacramento s/n, E04120 La Cañada de San Urbano, Almería, Spain \\ d CIEMAT-Plataforma Solar de Almería, Ctra. de Senés, km. 4.5, E04200 Tabernas, Almería, Spain
}

\section{A R T I C L E I N F O}

\section{Article history:}

Received 24 October 2012

Accepted 28 June 2013

Available online

\section{Keywords:}

Volumetric receiver

Heat transfer

Computational Fluid Dynamics (CFD)

2D thermal simulation

\begin{abstract}
A B S T R A C T
The thermal evaluation of different absorber configurations for a volumetric solar receiver designed for a solar furnace has been carried out by means of commercial Computational Fluid Dynamics (CFD) software in a 2D numerical model. Simulation results for proposed configurations depending on the porosity are discussed and compared to find the optimum configuration for which flow instabilities and thermal stresses are minimized and higher efficiencies are reached. The results obtained from the comparison of air velocity and thermal profiles at the absorber outlet propose a gradual-porosity configuration as an alternative to a previous design of a porous silicon-carbide honeycomb structure in order to heat an air stream up to temperatures suited for several high-temperature industrial processes.
\end{abstract}

(c) 2013 Elsevier Ltd. All rights reserved.

\section{Introduction}

Several studies have been carried out to analyze the behaviour of volumetric solar receivers in order to produce thermal energy for engines [1] or chemical processes [2-4].

Volumetric solar receivers are thermal systems, in which concentrated-solar radiation is absorbed on the surface of a material, which transfers the heat to a working fluid. The absorbed heat is transferred when the fluid passes through a porous medium. The characterization and design of these media have been carried out by several authors, in order to obtain efficient solar thermal receivers which have had a significant interest in the conversion from solar to electrical power and heating applications during the past 30 years.

One of this studies tested a porous ceramic absorber, consisting of a $92 \%$-alumina foam, which heated an air stream up to $730{ }^{\circ} \mathrm{C}$ with a solar flux of up to $824 \mathrm{~kW} / \mathrm{m}^{2}$ [5]. Another design considered a ceramic foil receiver covered by a matrix of square channels of quartz glass, in order to absorb the thermal radiation emitted by the ceramic part of the receiver. The outlet air reached temperatures up to $1000{ }^{\circ} \mathrm{C}$ and efficiency improvements up to $10 \%$ in comparison with the thermal efficiency of $68.5 \%$ obtained from a ceramic foil receiver without quartz glass structure [6].

\footnotetext{
* Corresponding author. Tel.: +34 950387800x839; fax: +34 950365300.

E-mail addresses: mariaisabel.roldan@psa.es,_mabel_roldan@yahoo.es (M.I. Roldán).
}

The concept of a multi-cavity volumetric solar receiver was regarded because of its higher efficiency and economy compared to the single cavity receiver. The absorber was based on a pack of small ceramic cavities which absorbed the high solar flux reflected from an array of mirrors [7,8].

Furthermore, volumetric solar receivers have required new materials and designs highly resistant to thermal stresses. One example was the "Porcupine", which endured a concentrated-solar flux of up to $4 \mathrm{MW} / \mathrm{m}^{2}$ reaching air temperatures of up to $940{ }^{\circ} \mathrm{C}$ [9].

High performance absorbers are damaged by local overheating due to inhomogeneous flux distributions and flow instabilities. Therefore, an optimized combination of geometrical properties as well as thermal conductivity and heat-transfer parameters has to be selected in order to achieve both high efficiencies and reliable operation. For this purpose, several methodologies have been developed to determine the thermal properties and behaviour of porous materials [10-12].

Both the analysis of these thermal properties and absorberbehaviour predictions have been carried out by Computational Fluid Dynamics (CFD). One of this analysis described the impact of several geometrical properties of the absorber and the influence of the air injection system used [13]. Furthermore, the pressure drop in ceramic foams for solar air receiver applications was analyzed by experimental and numerical studies, obtaining a modified Darcy relationship [14].

On the other hand, heat-transfer simulations were developed for different absorber configurations using CFD in order to achieve 


\begin{tabular}{|c|c|c|c|}
\hline \multicolumn{2}{|c|}{ Nomenclature } & $y$ & position in axis $y, \mathrm{~m}$ \\
\hline & & \multicolumn{2}{|c|}{ Greek symbols } \\
\hline \multicolumn{2}{|c|}{ Variable, description, unit } & $\rho$ & density, $\mathrm{kg} / \mathrm{m}^{3}$ \\
\hline$B$ & matrix of the momentum source term & $\lambda$ & thermal conductivity, $\mathrm{W} / \mathrm{m} \mathrm{K}$ \\
\hline C & correction coefficient dimensionless & $\mu$ & viscosity, $\mathrm{kg} / \mathrm{m} \mathrm{s}$ \\
\hline$c_{\mathrm{p}}$ & specific heat capacity, J/kg K & $\tau$ & stress tensor, $\mathrm{N} / \mathrm{m}^{2}$ \\
\hline$D$ & matrix of the momentum source term & $\Delta$ & variation in a property \\
\hline$E$ & specific energy, J/kg & $\eta$ & thermal efficiency, \% \\
\hline$F$ & external body force, $\mathrm{N}$ & $\xi$ & optical extinction coefficient, $\mathrm{m}^{-1}$ \\
\hline$F_{\text {Peak }}$ & irradiance peak, $\mathrm{W} / \mathrm{m}^{2}$ & $\theta$ & angle between the edges of the mesh element, ${ }^{\circ}$ \\
\hline & gravitational acceleration, $\mathrm{m} / \mathrm{s}^{2}$ & $\sigma_{x}$ & ellipticity factor, $\mathrm{m}$ \\
\hline$h$ & sensible enthalpy, $\mathrm{J} / \mathrm{kg}$ & & \\
\hline$I$ & intensity of the solar radiation, $\mathrm{W} / \mathrm{m}^{3}$ & \multicolumn{2}{|c|}{ Subscripts } \\
\hline$I_{0}$ & initial intensity of the solar radiation, $\mathrm{W} / \mathrm{m}^{3}$ & conv & convective \\
\hline$I_{\mathrm{SO}}$ & superficial heat source, $\mathrm{W} / \mathrm{m}^{2}$ & eff & effective \\
\hline$J$ & diffusion flux, $\mathrm{kg} / \mathrm{s} \mathrm{m}^{2}$ & eq & equilateral \\
\hline$k$ & thermal conductivity, $\mathrm{W} / \mathrm{m} \mathrm{K}$ & $\mathrm{f}$ & fluid \\
\hline$K_{1}$ & permeability, $\mathrm{m}^{2}$ & $\mathrm{~h}$ & heat \\
\hline$K_{2}$ & inertial coefficient, $\mathrm{m}^{-1}$ & $i$ & $i$ th \\
\hline$l$ & length of the absorber material, m & in & inlet \\
\hline$m$ & mass flow, kg/s & $j$ & species \\
\hline$p$ & static pressure, $\mathrm{N} / \mathrm{m}^{2}$ & $\mathrm{~m}$ & mass \\
\hline$Q$ & heat, W & mag & magnitude \\
\hline$Q_{\text {EAS }}$ & equiangle skew dimensionless & $\max$ & maximum \\
\hline$S$ & source term & $\min$ & minimum \\
\hline$T$ & temperature, $\mathrm{K}$ & out & outlet \\
\hline$t$ & time, $\mathrm{s}$ & rec & receiver \\
\hline$u$ & kinematic viscosity, $\mathrm{m}^{2} / \mathrm{s}$ & s & solid \\
\hline$v$ & velocity, $\mathrm{m} / \mathrm{s}$ & tot & total \\
\hline$x$ & position in axis $x, \mathrm{~m}$ & & \\
\hline
\end{tabular}

high performance in volumetric solar receivers. The convective heat transfer between the air flow and ceramic foam was numerically studied to compute the local heat-transfer coefficient [15-17].

In addition, another model was developed to take into account the radiative heat transfer due to the absorption of the concentrated-solar radiation by the absorber and the radiation transport in the media. The pressure drop was included together with the interfacial heat transfer between the flowing fluid and the porous material in the same model. The results illustrated that the thermal non-equilibrium phenomena are locally important [18], because they are present when there is a thermal gradient in the porous material. In that case, the viscosity of the air changes with the temperature together with permeability properties, and the homogeneity of the air flow is significantly affected by the permeability variation. Thus, the permeability in hot channels is lower, leading to its hot blocking. As a consequence, this model allowed the optimization of the volumetricair-receiver design.

Comparisons between different porous materials have been carried out, obtaining a significantly higher efficiency for a doublelayer foam material than a screen-printed porous silicon-carbide one. Porous materials with a parallel channel geometry with thin walls showed disadvantageous permeability properties. These printed-material properties can be significantly changed by applying a new manufacturing process and modifying the channel geometry and porosity [19].

Other volumetric-receiver concepts were studied in the past by other authors using an analytical model to guide the experimental design of volumetric flow receivers. This type consists of nanoparticles suspended in a heat-transfer fluid, in order to absorb the incoming radiation and to efficiently store the thermal energy in the fluid volume $[20,21]$.

Numerical studies allow analyzing new receiver configurations such as gradual-porosity designs which have a difficult and expensive manufacturing. Therefore the achievement of numerical methodologies which reproduce the thermal behaviour of these porous materials are necessary to select the best configuration and, as a consequence, to develop an appropriate manufacturing process.

This need has been satisfied by this study, because a new methodology of simulation for gradual-porosity receivers has been developed. This type of volumetric receivers are made up of various parts with a different porosity and the prediction of its thermal behaviour is a valuable new information, which has been obtained and added to the existing pool of knowledge concerning the volumetric-receiver design. For instance, the variation of geometric parameters influences the thermal efficiency of the porous structure. This behaviour can be analyzed using the numerical model developed.

Simulation results from gradual- and constant-porosity configurations have been compared in this study, where CFD has been used to optimize the absorber configuration of an open volumetric receiver installed at the Plataforma Solar de Almería's Solar Furnace (PSA Solar Furnace [22]). Therefore, a heat-transfer model has been carried out to predict the absorber behaviour of proposed configurations which use the same ceramic material. The porous material tested consisted of a silicon-carbide honeycomb structure by which an air stream is heated up to temperatures required in several hightemperature industrial processes. 


\section{Methodology}

\subsection{Procedure}

This study carries out the thermal and fluid dynamic analysis of different absorber configurations included in a volumetric solar receiver designed for the PSA Solar Furnace [1,23]. A commercial CFD software (Fluent) has been used for the study of the absorber configurations proposed, implementing a two-dimensional fluid model. The absorber receives concentrated-solar radiation. An air stream, that passes through this material, is heated in order to supply the energy of the process.

Simulations of different absorber configurations have been proposed to find the optimal one by which the highest temperatures are reached in the air stream, preserving the porous-medium properties. These configurations are proposed for the same absorber material (silicon carbide) and they are divided into three groups: one considers three different constant porosities $(0.48,0.64$, and 0.78$)$, the other group takes into account a gradual-porosity variation in the radial direction using the previous three porosities in increasing and decreasing order, and the last group includes a gradual configuration according to the absorber depth $(6 \mathrm{~cm})$ using also the increasing and decreasing order (see Table 1 ). In this case, the absorber has a circular shape with a radius of $10.5 \mathrm{~cm}$, similar to the radius of the concentrated-solar-radiation focus.

The simulation parameters have been evaluated by means of the reference values calculated for the configuration which was used in the experimental setup. This configuration consists of a siliconcarbide element of $10.5 \mathrm{~cm}$ in radius and $6 \mathrm{~cm}$ deep with a porosity of 0.64 (Fig. 1a). The fluid flow passes through square channels, distributed throughout the material, of $2 \mathrm{~mm}$ on each side and $6 \mathrm{~cm}$ in depth. The thickness of the wall, which separates the channels, is $0.5 \mathrm{~mm}$ (Fig. 1b).

In all cases, the absorber domain is defined in the model as a porous material. Therefore, the porosity of each configuration has been regarded instead of the channel shape. Thus, Fig. 2 represents the thermal balance for the domain selected, including the porosity effect on the radiation-intensity attenuation. In order to consider the energy absorbed, the volumetric-heat source has been calculated for each configuration and implemented by a user-defined function (UDF).

\subsection{Evaluation of the volumetric-heat source}

The volumetric-heat-source definition has been based on an exponential law, which is an approach of the radiation-intensity attenuation in the absorber material. The following equation describes this process $[11,12]$ :

$I(x, y)=I_{0} \cdot e^{-\xi \cdot y}$

where $I$ is the intensity of the solar radiation, which goes through the absorber depth, $\xi$ is the optical extinction coefficient, $I_{0}$ is the initial intensity of the solar radiation, $x$ is the position in $x$-axis direction, and $y$ is the position in $y$-axis direction (see Fig. 2).

Table 1

Proposed configurations.

\begin{tabular}{lllll}
\hline $\begin{array}{l}\text { Constant } \\
\text { porosities }\end{array}$ & \multicolumn{2}{l}{$\begin{array}{l}\text { Gradual porosities in } \\
\text { the radial direction }\end{array}$} & $\begin{array}{l}\text { Gradual porosities according } \\
\text { to depth }\end{array}$ \\
\hline 0.48 & 0.48 & $0-3.5 \mathrm{~cm}$ & 0.48 & $0-2 \mathrm{~cm}$ \\
& 0.64 & $3.5-7 \mathrm{~cm}$ & 0.64 & $2-4 \mathrm{~cm}$ \\
0.64 & 0.78 & $7-10.5 \mathrm{~cm}$ & 0.78 & $4-6 \mathrm{~cm}$ \\
& 0.78 & $0-3.5 \mathrm{~cm}$ & 0.78 & $0-2 \mathrm{~cm}$ \\
0.78 & 0.64 & $3.5-7 \mathrm{~cm}$ & 0.64 & $2-4 \mathrm{~cm}$ \\
& 0.48 & $7-10.5 \mathrm{~cm}$ & 0.48 & $4-6 \mathrm{~cm}$ \\
\hline
\end{tabular}

a)

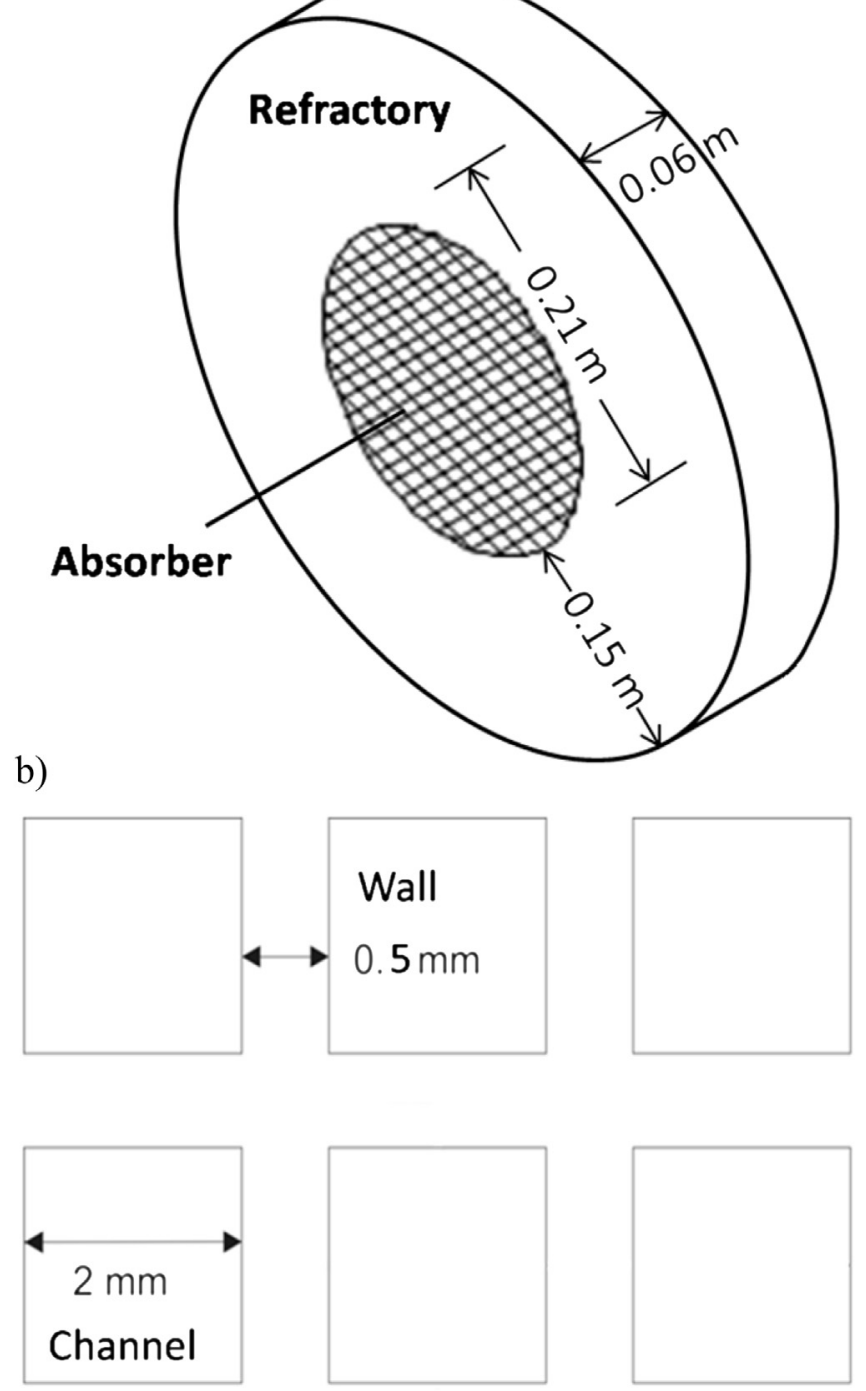

Fig. 1. Description of the volumetric solar receiver: a) module dimensions; b) absorber dimensions.

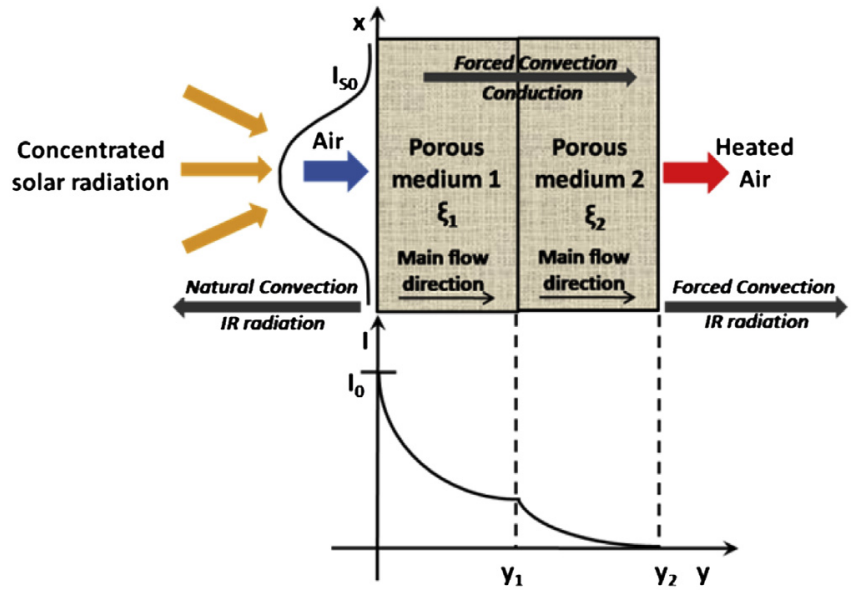

Fig. 2. Thermal balance sketch. 
$I_{0}$ depends on the superficial heat source $\left(I_{\mathrm{s} 0}\right)$, which is evaluated from the optical properties of the PSA Solar Furnace. The theoretical model used to define $I_{\mathrm{S} 0}$ includes a Gaussian distribution which depends on the $x$-axis position $(x)$ for 2D numerical simulation [24]:

$I_{\mathrm{S} 0}(x)=F_{\text {Peak }} \cdot \exp \left(\frac{-1}{2} \cdot\left(\frac{x^{2}}{\sigma_{x}^{2}}\right)\right)$

where $F_{\text {Peak }}$ is the irradiance peak, $\sigma_{x}$ is the ellipticity factor, which takes into account the Gaussian-function shape. $F_{\text {Peak }}$ and $\sigma_{x}$ are evaluated from a previous analysis of the CCD-camera images obtained from the heat-flux measurements in different positions starting from the focal position $[25,26]$. The irradiance-peak estimation includes all the parameters which affect on the amount of solar energy concentrated by the facility. The theoretical prediction of the heat-flux distribution on the absorber surface has been compared with experimental measurements obtained from infrared images recorded during the time considered as steady-state conditions in the test.

In this case, ideal steady-state conditions cannot be obtained [27] and a quasi-steady state has been selected, which includes the images with a maximum-temperature variation less than $5 \%$ in the last

a)

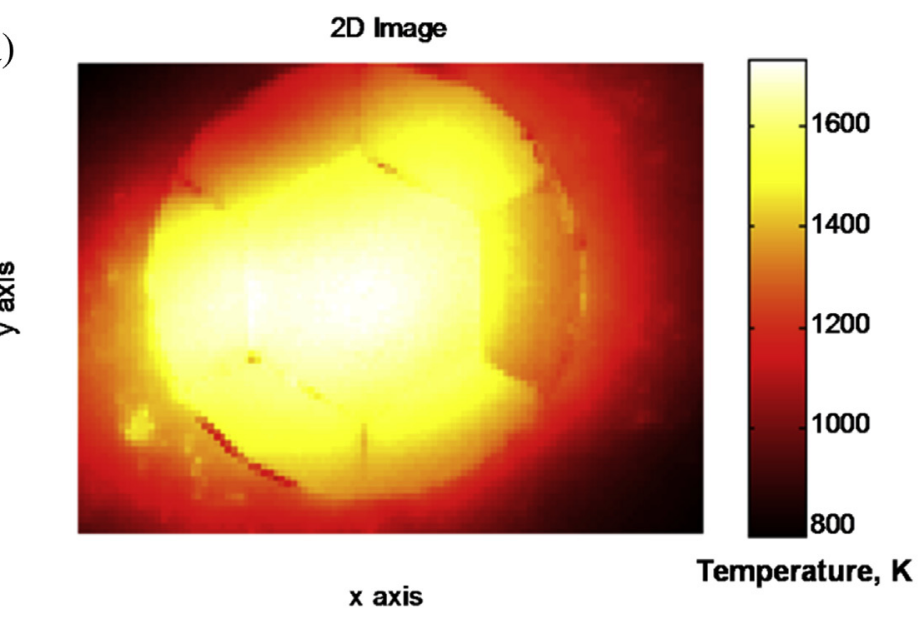

b)

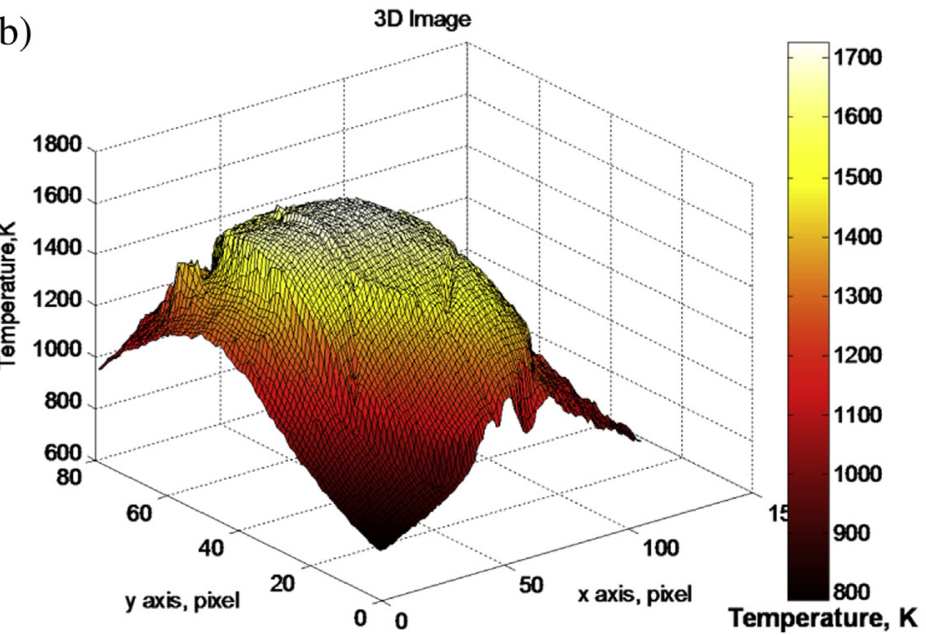

c)

Thermal-profile comparison

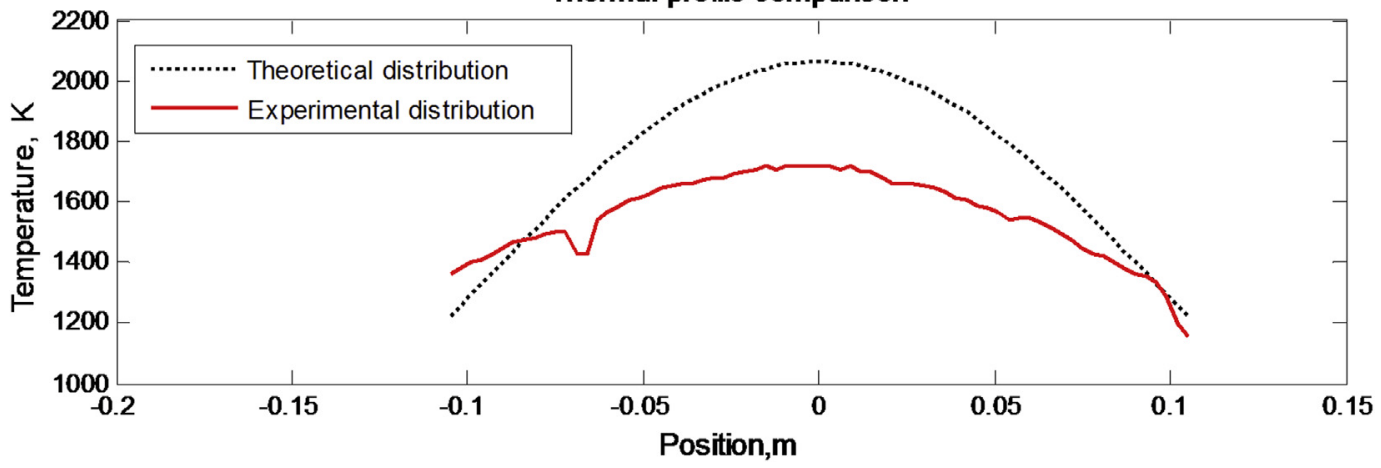

Fig. 3. Analysis of infrared images: a) 2D pixel-intensity calibration by Matlab ${ }^{\circledR}$; b) 3D pixel-intensity calibration; c) thermal profile. 

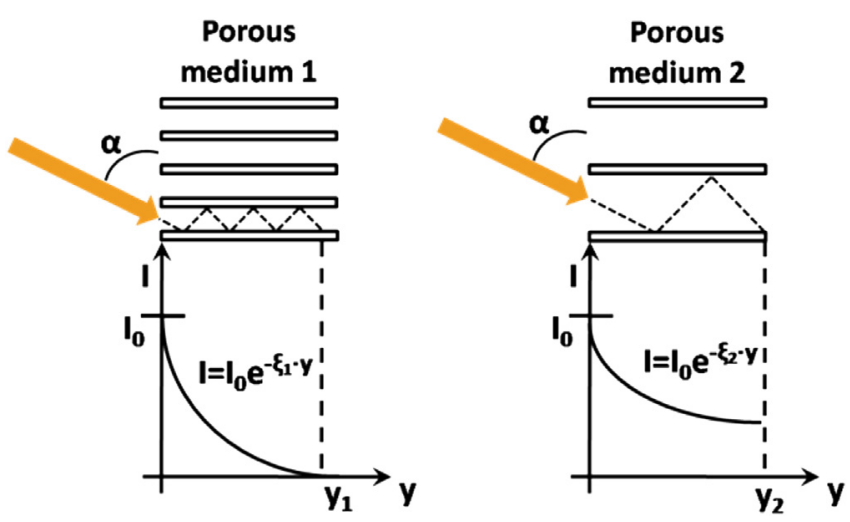

Fig. 4. Radiation-intensity attenuation depending on the porosity.

15-min images recorded during the test. The image analysis consists of a thermal-profile evaluation by means of a pixel-intensity calibration (Fig. 3a and b) whose central thermal profile is compared with the theoretical thermal profile, which comes from considering Stefan-Boltzmann law [28] for the theoretical heat-flux distribution (Fig. 3c). The difference between both profiles is because, on the one hand, $I(x, y)$ evaluation does not consider the thermal losses due to the convection between the absorber surface and ambient air, and, on the other hand, the absorption properties have not been taken into account in the definition of the solar-radiation intensity absorbed by the porous material. These properties depend on its conductivity. Therefore, these factors have been regarded by means of a correction coefficient $(C)$ defined as the average difference.

Consequently, the general equation considered for the volumetric-heat source is:

$I(x, y)=\left(F_{\text {Peak }} \cdot \exp \left(\frac{-1}{2} \cdot\left(\frac{x^{2}}{\sigma_{x}^{2}}\right)\right)\right) \cdot C \cdot \xi \cdot e^{-\xi \cdot y}$

where:

$I_{0}=I_{\mathrm{S} 0} \cdot \xi\left(\mathrm{W} / \mathrm{m}^{3}\right)$

and $\xi$ is estimated for each different porosity proposed from the reference configuration, because the radiation-intensity attenuation depends on the material porosity, as it is shown in Fig. 4. This reference value was evaluated at central position $(0,0)$ with $F_{\text {Peak }}$

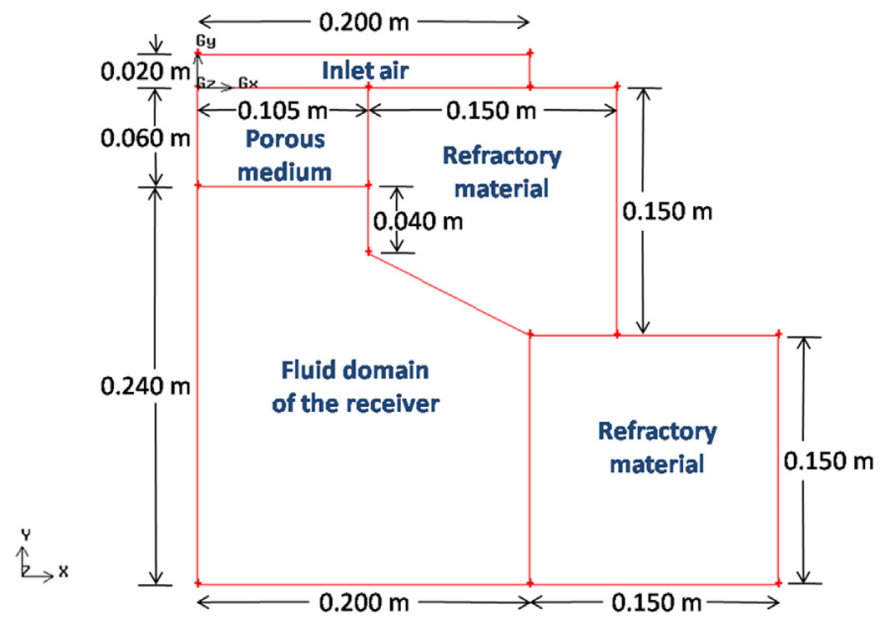

Fig. 5. Description of the solution domain. and $C$ obtained from the experimental conditions of a selected test $\left(953,500 \mathrm{~W} / \mathrm{m}^{2}\right.$ and 0.849 , respectively). The range of the volumetric-heat flux $\left(10^{07} \mathrm{~W} / \mathrm{m}^{3}\right)$ was obtained from a previous study [29], which considers an absorber behaviour similar to the one used in the current analysis. In this case, the exponential functions are equivalent to 1 because $y=0$ in the selected position, and $\xi$ is evaluated from equation (4) $\left(12.35 \mathrm{~m}^{-1}\right)$.

\subsubsection{Constant porosity}

The volumetric-heat source for the reference configuration (constant porosity of 0.64 ) is defined by the following equation:

$$
\begin{aligned}
I(x, y)= & \left(953,500 \cdot \exp \left(\frac{-1}{2} \cdot\left(\frac{x^{2}}{0.0724^{2}}\right)\right)\right) \\
& \cdot 0.849 \cdot 12.35 \cdot e^{-12.35 \cdot y}
\end{aligned}
$$

The extinction coefficients for the other porosities considered $(0.48,0.78)$ were calculated from the comparison between the heat-transfer coefficient of the reference porosity and the others obtained for 0.48 and 0.78 . These heat-transfer coefficients were

a)

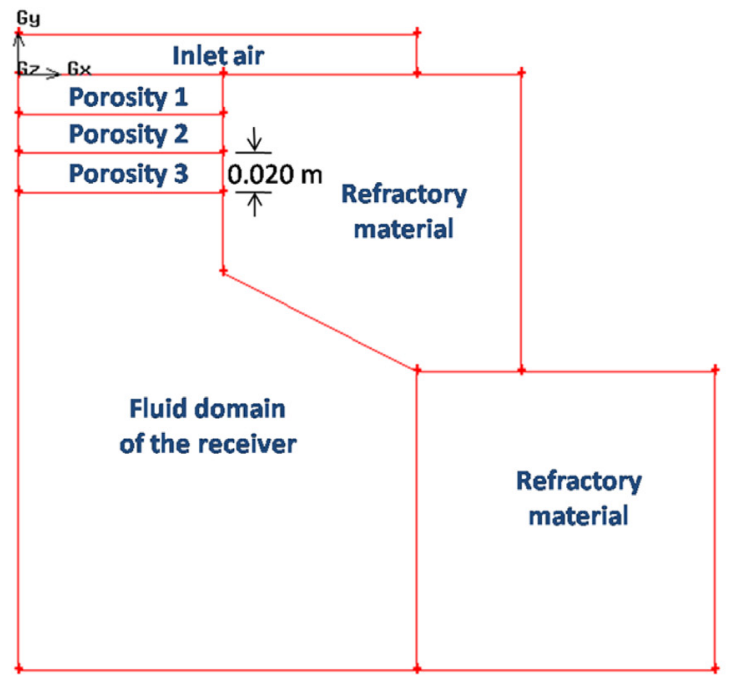

b)

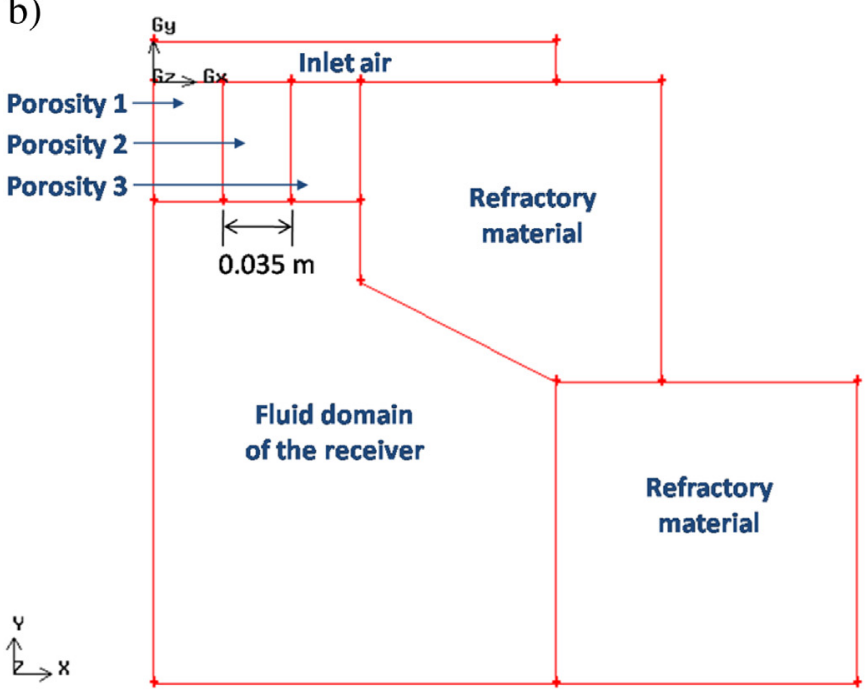

Fig. 6. Sub-domains considered in gradual configurations: a) gradual porosity according to depth; b) gradual porosity in the radial direction. 
Table 2

Mesh quality evaluation.

\begin{tabular}{lllr}
\hline$Q_{\text {EAS }}$ range & Mesh quality & Elements & $\%$ \\
\hline $0-0.1$ & Excellent & 8309 & 76.45 \\
$0.1-0.2$ & Excellent & 1384 & 12.73 \\
$0.2-0.25$ & Excellent & 720 & 6.62 \\
$0.25-0.302$ & Good & 455 & 4.19 \\
\hline
\end{tabular}

estimated from the simulations under the same conditions as the reference-porosity simulation. In all cases, the volumetric-heatsource equation was implemented as a UDF.

\subsubsection{Gradual porosity in the radial direction}

For radial direction, the porous domain consists of three subdomains located as concentric rings. Consequently, the Gaussian function cannot be used because the CFD software would interpret three maximum temperatures, one for each sub-domain, due to the definition of the volumetric energy source, which must be independent for each one. To avoid that, an average value substitutes the Gaussian function in the equation of the volumetricheat source $\left(456,000 \mathrm{~W} / \mathrm{m}^{2}\right)$ bringing as a result the following expression:

$$
I(y)=456,000 \cdot \xi \cdot e^{-\xi \cdot y}
$$

$\xi$ is replaced in equation (6) by the value obtained for each porosity and a UDF library has been created in order to simultaneously include the three equations. For that purpose, an external programme was developed using the own software code and implemented by means of a compiler.

\subsubsection{Gradual porosity according to depth}

In order to integrate into the model the heat-source equation for each porosity, the porous domain has been divided into three subdomains. Each one includes equation (6) with a different value of the extinction coefficient (Table 3 ). This means that the simulation requires to use three UDFs at the same time, therefore the UDF library created for gradual porosity in the radial direction has been implemented. Considering the average value for the superficial heat source allows making a comparison between both gradual configurations.

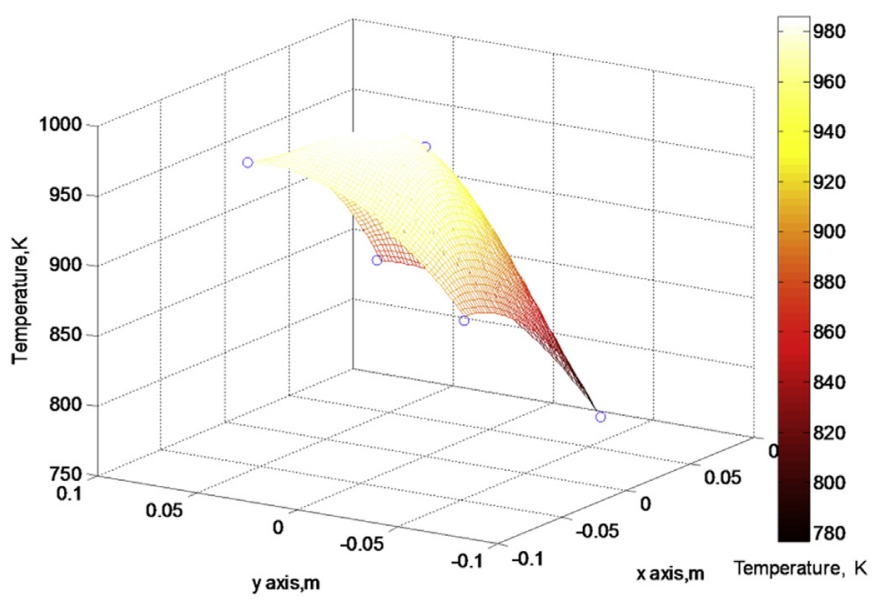

Fig. 7. Interpolation of the experimental measurements at the absorber outlet.

\subsection{Simulation model}

\subsubsection{Numerical modelling}

Conservation laws (conservation of mass, momentum, and energy) determine the fluid dynamics behaviour [30]. Therefore the CFD model developed requires solving the continuity (7), momentum (8) [31] and energy (9) [32] equations described for fluid medium by the following expressions:

$\frac{\partial \rho}{\partial t}+\nabla(\rho \cdot \vec{v})=S_{\mathrm{m}}$

$\frac{\partial}{\partial t}(\rho \cdot \vec{v})+\nabla(\rho \cdot \vec{v} \cdot \vec{v})=-\nabla p+\nabla \cdot(\overline{\bar{\tau}})+\rho \cdot \vec{g}+\vec{F}$

$\frac{\partial}{\partial t}(\rho \cdot E)+\nabla \cdot(\vec{v}(\rho E+p))=\nabla \cdot\left(k_{\mathrm{eff}} \nabla T-\sum_{j} h_{j} \cdot \vec{J}_{j}+\left(\overline{\bar{\tau}}_{\mathrm{eff}} \cdot \vec{v}\right)\right)+S_{\mathrm{h}}$

where $\rho$ is the density of the fluid, $t$ is elapsed time, $\vec{v}$ is the velocity vector with respect to the coordinate system, $S_{\mathrm{m}}$ is the mass source, $p$ is the static pressure, $\overline{\bar{\tau}}$ is the stress tensor, $\rho \cdot \vec{g}$ is the gravitational body force, $\vec{F}$ is the external body force, $E$ is the energy transfer $\left(E=h-p / \rho+v^{2} / 2\right), k_{\text {eff }}$ is the effective conductivity which includes the turbulence thermal conductivity, $h_{j}$ is the enthalpy of

a)

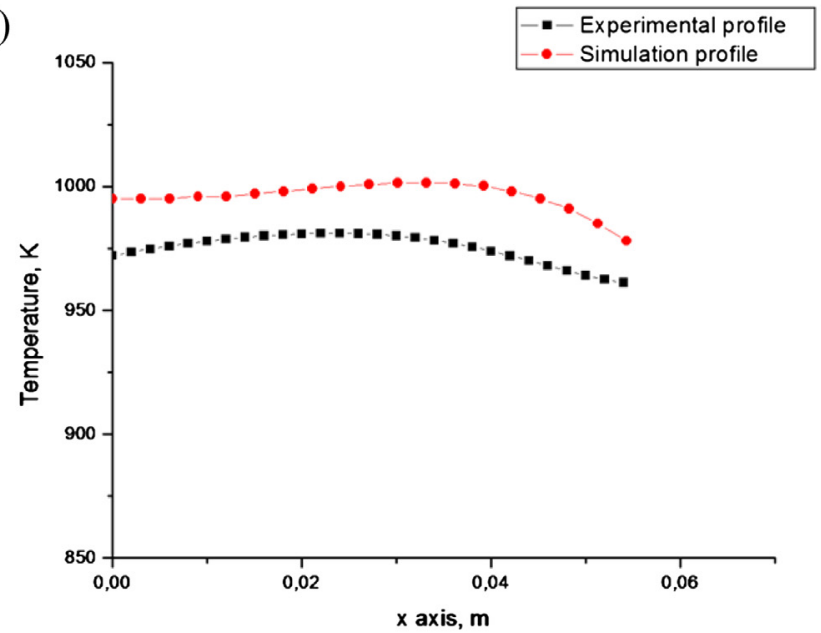

b)

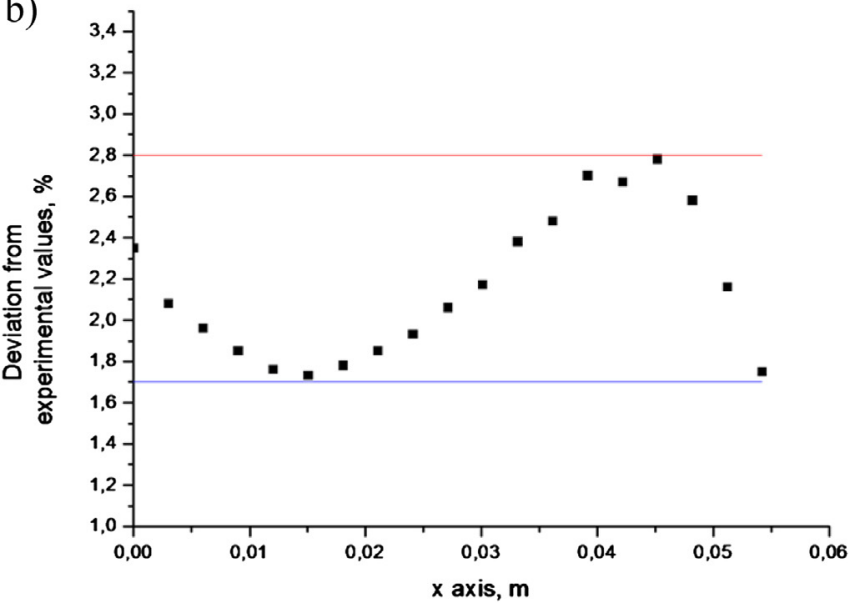

Fig. 8. Comparison between experimental measurements and simulation results: a) thermal profiles; b) deviation from the experimental values. 
species $j, \overrightarrow{J_{j}}$ is the diffusion flux of species $j, \overline{\bar{\tau}}_{\text {eff }}$ is the viscous stress tensor, and $S_{\mathrm{h}}$ is the volumetric-heat source. These general equations are regarded in two dimensions and, in this case, the air is the only species involved in the fluid medium.

The porous-medium model takes into account the solid influence on the fluid and it requires to include the solid effect in the energy equation and to consider an additional momentum source term. Therefore, equation (8) implements for the porous medium a source term (equation (10)) composed of two parts: a viscous loss term and an inertial loss term.

$S_{i}=-\left(\sum_{j=1}^{2} D_{i j} \mu v_{j}+\sum_{j=1}^{2} B_{i j} \frac{1}{2} \rho v_{\operatorname{mag}} v_{j}\right)$

where $i$ and $j$ take into account the coordinate directions of the model ( $x$ or $y$ ), $S_{i}$ is the source term for the $i$ th ( $x$ or $y$ directions) momentum equation, $v_{\mathrm{mag}}$ is the velocity magnitude, and $D$ and $B$ are prescribed matrices [33].

The energy equation for the porous medium is described by the following expression:

$$
\begin{aligned}
& \frac{\partial}{\partial t}\left(\gamma \rho_{\mathrm{f}} E_{\mathrm{f}}+(1-\gamma) \rho_{\mathrm{s}} E_{\mathrm{s}}\right)+\nabla \cdot\left(\vec{v}\left(\rho_{\mathrm{f}} E_{\mathrm{f}}+p\right)\right) \\
& \quad=\nabla \cdot\left(k_{\mathrm{eff}} \nabla T-\sum_{j} h_{j} \cdot \vec{J}_{j}+\left(\overline{\bar{\tau}}_{\mathrm{eff}} \cdot \vec{v}\right)\right)+S_{\mathrm{f}}^{h}
\end{aligned}
$$

where $\gamma$ is the porosity of the medium, $E_{\mathrm{f}}$ is the total fluid energy, $E_{\mathrm{s}}$ is the total solid energy, $S_{\mathrm{f}}{ }_{\mathrm{f}}$ is the fluid enthalpy source term, and $k_{\text {eff }}$ is defined by the following equation:

$k_{\mathrm{eff}}=\gamma \cdot k_{\mathrm{f}}+(1-\gamma) \cdot k_{\mathrm{s}}$

where $k_{\mathrm{f}}$ is the fluid-phase thermal conductivity, including the turbulent contribution, and $k_{\mathrm{s}}$ is the solid-medium thermal conductivity [33].

The flow rate forced by a blower has been evaluated at the moment considered as a quasi-steady state, in order to select the flow regimes present in the solution domain shown in Fig. 5. Laminar and transitional flow regimes have been defined according to the sub-domain considered (Section 2.3.2).

In the absorber domain (porous medium), the flow regime has been considered laminar, but, in the fluid domain of the receiver, a viscous model has been used. The model selected was renormalization-group (RNG) $\kappa-\varepsilon$ model because it includes an analytically-derived differential formula for effective viscosity that accounts for low-Reynolds-number effects [34]. The near-wall region was calculated by the 'wall function' approach, in which semiempirical formulae are used to obtain the viscosity between the wall and the turbulent region [35].

\subsubsection{Geometry definition and mesh design}

Fig. 5 describes the solution domain, in which the 2D symmetrical part of the receiver inlet is represented. The $3 \mathrm{D}$ domain can be obtained from the revolution of the symmetry axis because the receiver presents a cylindrical configuration. A two-dimensional model has been considered in this case, to define a model which is as simply as possible and able to predict the hydraulic and thermal behaviour for gradual-porosity receivers, while minimizing the computational requirements in comparison with a complex and time-consuming model. This fact allows making the reproducibility of the experimental cases easier, obtaining results in a shorter time.

Several sub-domains have been defined in the solution domain (Fig. 5), in order to select the appropriate conservation-law equations which describe the physical phenomena occurred in each section. The sub-domains were selected depending on the boundary conditions, and the regions obtained were: inlet air which defines the ambient conditions and air flow, two refractory-material domains to include the influence of the insulation in the model, porous medium which determines the heat transfer from the absorber to the air, and the heated air of the receiver which supplies the energy for industrial thermal processes which require high-temperature conditions, mainly for material treatments.

In the constant-porosity configuration, the porous medium is regarded as a single sub-domain. However, in the case of the gradual porosity, the absorber domain has been divided into different sub-domains depending on the porosity (see Fig. 6).

The grid for this solution domain was created by a commercial mesh generator (Gambit), which has discretized the selected domain with a 2D structured mesh of quadrilateral elements. The equiangle skew ( $Q_{\text {EAS }}$ ) is the mesh quality-type specification used to evaluate the mesh size. The $Q_{E A S}$ is a normalized measure of skewness defined by Ref. [36]:

$Q_{\mathrm{EAS}}=\max \left\{\frac{\theta_{\mathrm{max}}-\theta_{\mathrm{eq}}}{180-\theta_{\mathrm{eq}}}, \frac{\theta_{\mathrm{eq}}-\theta_{\mathrm{min}}}{\theta_{\mathrm{eq}}}\right\}$

where $\theta_{\max }$ and $\theta_{\min }$ are the maximum and minimum angles between the edges of the element, and $\theta_{\mathrm{eq}}$ is the characteristic angle corresponding to an equilateral cell of similar form $\left(\theta_{\mathrm{eq}}=90^{\circ}\right.$ for quadrilateral elements).

By definition $0 \leq Q_{\mathrm{EAS}} \leq 1$, and the mesh element is equilateral when $Q_{E A S}$ is 0 , having a poor shape when $Q_{E A S}$ is 1 . The analysis of the mesh quality is shown in Table 2, where $100 \%$ of the cells are included between the values of 0 and 0.5 . This range represents a good quality according to the relationship between $Q_{E A S}$ and mesh quality presented by Ref. [36], and, in general, it is considered that 2D high-quality meshes contain elements that possess average $Q_{E A S}$ values of 0.1 [36]. In this case, the average $Q_{\text {EAS }}$ is 0.13 , and it is close to the value which determines the high-quality mesh. Therefore, this mesh analysis has led to select the designed grid for the simulations.

\subsubsection{Operating conditions and physical properties}

The experimental configuration has a constant porosity of 0.64 and it is assumed that the fluid (air) is under steady-state flow condition, and the blower of the volumetric receiver produces a flow rate whose value is evaluated for the quasi-steady state condition $(0.013 \mathrm{~kg} / \mathrm{s})$. The operating pressure considered for the CFD model has been set to the value of $101,325 \mathrm{~Pa}$. The gravitational force can be neglected due to the low density of the fluid and the

Table 3

\begin{tabular}{|c|c|c|c|c|c|}
\hline Porosity & Average of the heat-transfer coefficient, $\mathrm{W} / \mathrm{m}^{2} \mathrm{~K}$ & Deviation \% & Correction factor & Volumetric-heat source, $\mathrm{W} / \mathrm{m}^{3}$ & Extinction factor, $\mathrm{m}^{-1}$ \\
\hline 0.48 & 89.04 & -10.44 & 0.89 & $8.92 \cdot 10^{06}$ & 11.02 \\
\hline 0.64 & 99.42 & Reference & Reference & $10^{07}$ & 12.35 \\
\hline 0.78 & 105.81 & 6.43 & 1.06 & $1.06 \cdot 10^{07}$ & 13.15 \\
\hline
\end{tabular}

Extinction-coefficient evaluation of the new porosities considered. 
horizontal prototype position, and a mass source has not been considered because it is referred to the mass added to the continuous phase from a dispersed second phase (in this case, there is only one phase) and any user-defined sources [37].

a)

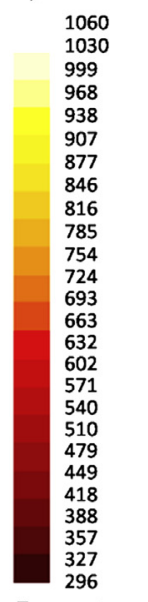

Temperature, $\mathbf{K}$

b)

1060
1030
999
968
938
907
877
846
816
785
754
724
693
663
632
602
571
540
510
479
449
418
388
357
327
296

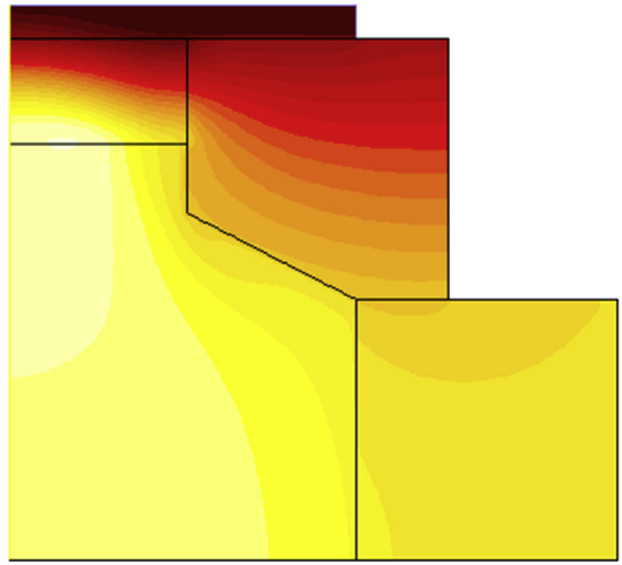

Temperature, $\mathrm{K}$

c)

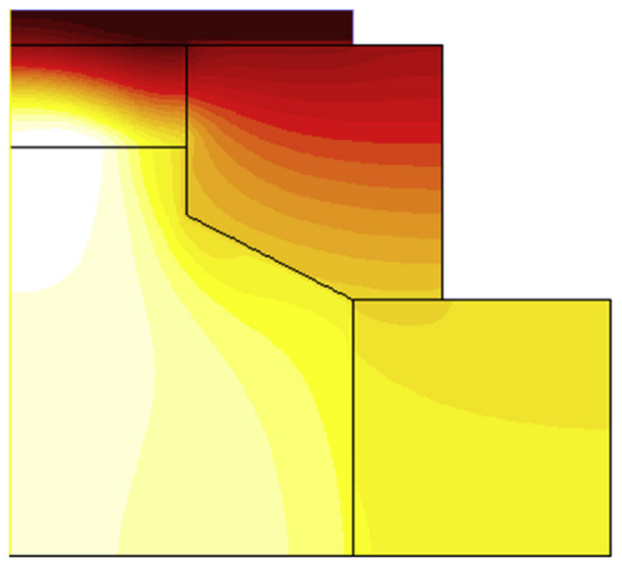

Temperature, $\mathrm{K}$
The thermophysical properties of the air stream have been defined for an average relative humidity of $43 \%$ at the temperature of $296 \mathrm{~K}$. These averaged conditions have been obtained from the measurement of ambient air during the test. The air properties can be described by the following equations, derived from the available moist air studies [38-44], in which the temperature must be considered in $\mathrm{K}$ :

$$
\begin{aligned}
& \rho_{\mathrm{f}}= 3.018 \cdot \exp (-0.00574 \cdot T) \\
&+0.8063 \cdot \exp (-0.0008381 \cdot T), \mathrm{kg} / \mathrm{m}^{3} \\
& \mu_{f}=\frac{1.458 \cdot 10^{-6} \cdot T^{3 / 2}}{T+110.4}, \mathrm{~kg} / \mathrm{ms} \\
& C_{\mathrm{pf}=} \\
& \quad-968 \cdot 10^{-10} \cdot T^{4}-8.332 \cdot 10^{-7} \cdot T^{3}+0.001187 \cdot T^{2} \\
& \\
& \lambda_{\mathrm{f}}= 9.354 \cdot 10^{-12} \cdot T^{3}-4.103 \cdot 10^{-08} \cdot T^{2}+9.986 \cdot 10^{-05} \cdot T \\
&+0.0005786, \mathrm{~W} / \mathrm{m} \mathrm{K}
\end{aligned}
$$

The thermophysical properties of the refractory and porous materials considered in the simulations were supplied by the data
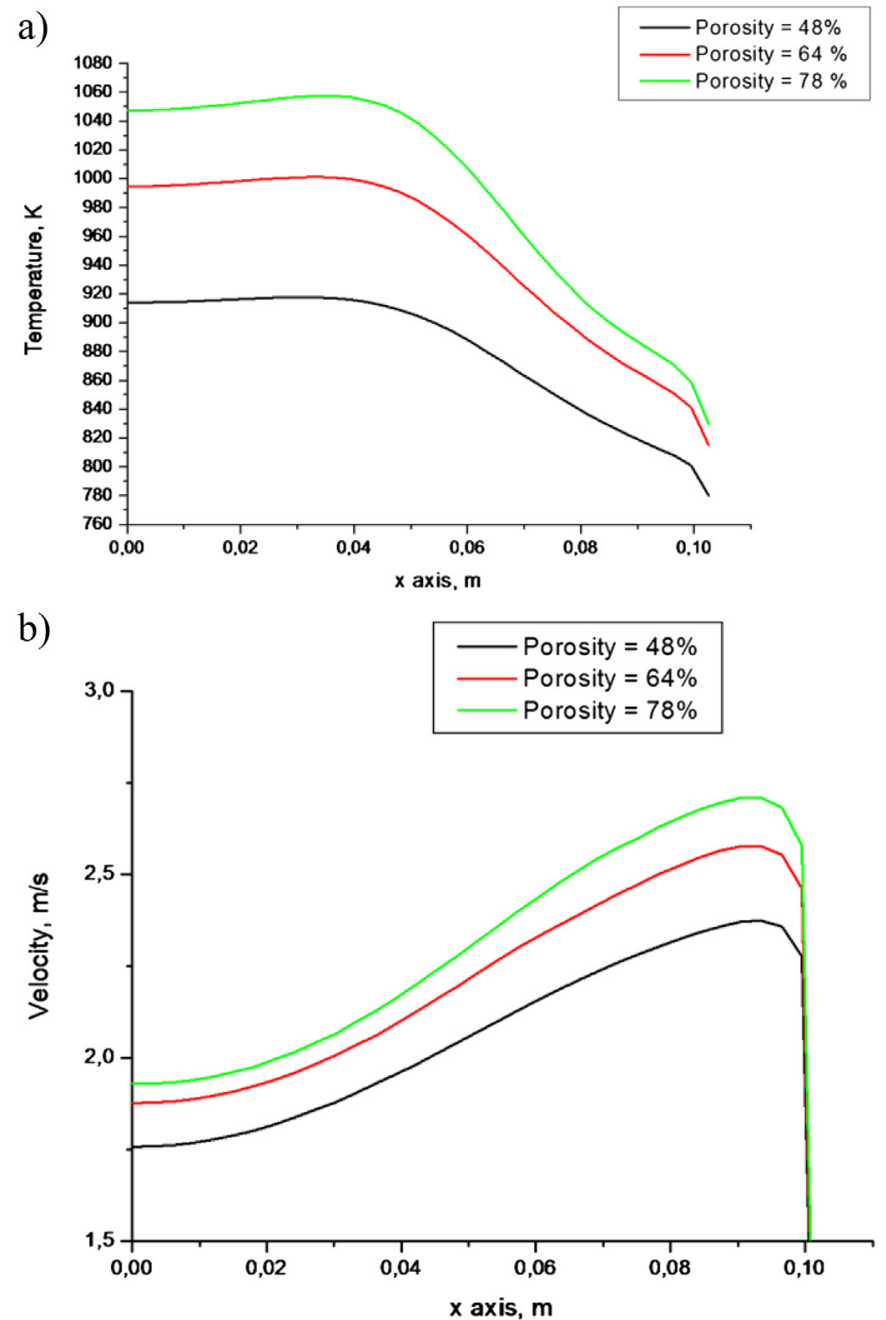

Fig. 10. Profiles at the porous-medium outlet: a) thermal profile; b) velocity profile. 
Table 4

Evaluation of the thermal efficiency considering an average value for the superficial heat source.

\begin{tabular}{lllllll}
\hline Configuration & Porosity & $T_{\text {in }}, \mathrm{K}$ & $T_{\text {out, }}, \mathrm{K}$ & $Q_{\text {conv }}, \mathrm{W}$ & $Q_{\mathrm{rec}}, \mathrm{W}$ & $\eta, \%$ \\
\hline Constant porosity & 0.48 & 378.9 & 621.6 & 21,083 & 43,959 & 47.96 \\
& 0.64 & 394.9 & 660.3 & 23,099 & 44,565 & 51.83 \\
& 0.78 & 406.4 & 683.1 & 24,118 & 44,840 & 53.79 \\
Gradual porosity in & Increasing & 434.8 & 791.5 & 28,702 & 43,183 & 66.47 \\
$\quad$ radial direction & Decreasing & 502.1 & 908.7 & 33,139 & 46,225 & 71.69 \\
Gradual porosity & Increasing & 384.4 & 756.2 & 32,730 & 44,287 & 73.90 \\
according to depth & Decreasing & 375.7 & 788.1 & 36,274 & 44,243 & 81.99 \\
\hline
\end{tabular}

sheets of each material [45]. The thermal conductivity was regarded for an average temperature, because the software assumes a constant thermal conductivity in thin walls.

The information of these materials is not available in the commercial CFD code, thus new-material definitions were implemented in the database, regarding the selected properties for each one.

\subsubsection{Boundary conditions}

The continuum zones defined in the solution domain were: porous medium, refractory material of the absorber module, inlet air, refractory material of the receiver, and the fluid of the receiver. In the case of gradual porosity, three different sub-domains have been considered in the porous medium, one per each porosity.

The porous-medium continuum zone includes the UDF of the volumetric-heat source, and the viscous loss term and the inertial loss term. These parameters have been evaluated experimentally by the measurement of the pressure loss depending on the air velocity when it passes through the absorber material. These two variables are related one to another by the Darcy's law in the Forchheimer extension for one direction (equation (18)), and the inverse of the denominator number $\left(K_{1}\right.$ and $\left.K_{2}\right)$, obtained from the fitting-function coefficients, evaluates the viscous loss term $\left(1.6 \cdot 10^{07} \mathrm{~m}^{-2}\right)$ and the inertial loss term $\left(52.63 \mathrm{~m}^{-1}\right)$ [12]. These parameters have been considered in the direction $(0,-1)$, and, in the direction $(1,0)$, a much greater value has been regarded $\left(10^{10} \mathrm{~m}^{-2}\right.$ and $1000 \mathrm{~m}^{-1}$, respectively) to enhance the fluid flow in the main direction $(0,-1)$.

$\frac{\Delta P}{l}=\frac{u \cdot \rho}{K_{1}} \cdot v+\frac{\rho}{K_{2}} \cdot v^{2}$

The walls, which delimit the porous medium, have a porousjump condition. This case requires to include the material permeability and the pressure-jump coefficient. The permeability is $6.11 \cdot 10^{08} \mathrm{~m}^{2}\left(K_{1}\right)$, and the pressure-jump was evaluated from the second coefficient of the fitting function for the Forchheimer's law $\left(104.73 \mathrm{~m}^{-1}\right)$ [11].

The velocity inlet condition was used in the inlet air domain and considers a velocity of $0.332 \mathrm{~m} / \mathrm{s}$. This velocity comes from the evaluation of the mass flow at ambient temperature in the quasisteady state selected. On the other hand, the direction of the flow was defined with the vector components $(0,-1)$ in the Cartesian coordinate system obtained from the mesh generator.
Furthermore, the walls connected with the insulating material, which has not been regarded in this simulation, have been defined as adiabatic walls.

A convective condition was defined in the outer wall, and the average heat-transfer coefficient because natural convection was fixed $\left(13.25 \mathrm{~W} / \mathrm{m}^{2} \mathrm{~K}\right)$ [46]. The inner wall of each domain was coupled with the remaining boundary zones.

\subsubsection{Solution method}

Simulations were performed by commercial CFD software, in which governing equations, operating conditions, material and fluid properties, and boundary conditions were selected.

The segregated steady-state solver was used to solve the governing equations, and variable residuals were monitored to ensure the convergence criterion, which were fixed at $10^{-6}$ for the energy, and at $10^{-3}$ as a minimum for the continuity and momentum variables.

The renormalization-group (RNG) $\kappa-\varepsilon$ turbulence-model constants were set to the software's default values. Furthermore, the pressure discretization scheme used was standard, and the algorithm for the pressure-velocity coupling chosen was simple.

The first order upwind is a convection-diffusion upwinding scheme which assumes that the cell-centre values of any field variable represent the cell-average value [47].

\section{Results and discussion}

\subsection{Validation}

In order to validate the simulation model, experimental air temperature at the porous-material outlet and simulation results at the same location were compared, considering the quasi-steady state of a test. That moment was selected using the Gaussian distribution which defines the superficial heat source (equation (2)). The reference absorber configuration of this test consisted of a constant porosity of 0.64 .

Fig. 7 shows the air thermal distribution at the absorber outlet, which comes from the spatial interpolation of the experimental measurements obtained from a programme developed by Matlab ${ }^{\circledR}$. These measurements correspond to the air temperature from five Type-K thermocouples Class I located on the same transversal surface at the absorber outlet (blue circles). As a result of the spatial interpolation from the experimental data, a temperature distribution surface was obtained. From this surface, a thermal profile in the $x$-axis direction has been obtained to make the comparison with the simulation thermal profile (Fig. 8a). The simulation-profile deviation from the experimental values is in the range of $1.7-2.8 \%$ (Fig. 8b). Therefore, the theoretical model used in the simulations can predict the absorber behaviour with a maximum deviation of $3 \%$ from the experimental values, including the deviation produced by the measuring instrument.

The Type-K thermocouples used have an accuracy of $\pm 1.5 \mathrm{~K}$ up to measurements of $648 \mathrm{~K}$, according to standard IEC 60584.2 (1982). For temperature ranges of $648-1273 \mathrm{~K}$, the accuracy

Table 5

Comparison between the gradual-porosity configurations considering an average value for the superficial heat source.

\begin{tabular}{|c|c|c|c|c|c|}
\hline Configuration & Porosity & $\begin{array}{l}\text { Maximum } \\
\text { temperature, } \mathrm{K}\end{array}$ & $\begin{array}{l}\text { Thermal gradient at } \\
\text { the absorber outlet, } \mathrm{K}\end{array}$ & $\begin{array}{l}\text { Minimum velocity at } \\
\text { the centre, } \mathrm{m} / \mathrm{s}\end{array}$ & $\begin{array}{l}\text { Velocity gradient at the } \\
\text { absorber outlet, m/s }\end{array}$ \\
\hline \multirow[t]{3}{*}{ Constant porosity } & 0.48 & 634.7 & 60.7 & 1.139 & 0.63 \\
\hline & 0.64 & 678.1 & 79.0 & 1.210 & 0.67 \\
\hline & 0.78 & 705.4 & 96.3 & 1.241 & 0.72 \\
\hline \multirow{2}{*}{$\begin{array}{l}\text { Gradual porosity } \\
\text { in radial direction }\end{array}$} & Increasing & 937.6 & 315.6 & 1.344 & 0.62 \\
\hline & Decreasing & 1323.1 & 758.6 & 1.349 & 0.71 \\
\hline \multirow{2}{*}{$\begin{array}{l}\text { Gradual porosity } \\
\text { according to depth }\end{array}$} & Increasing & 785.4 & 108.4 & 1.521 & 0.56 \\
\hline & Decreasing & 829.3 & 150.7 & 1.537 & 0.65 \\
\hline
\end{tabular}


a)

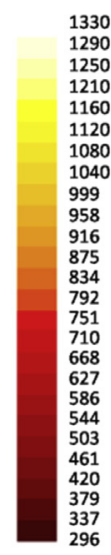

Temperature, $\mathrm{K}$

b)

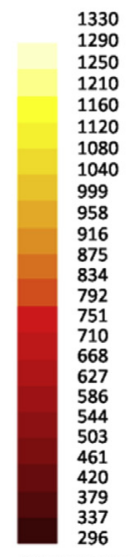

Temperature, $\mathrm{K}$
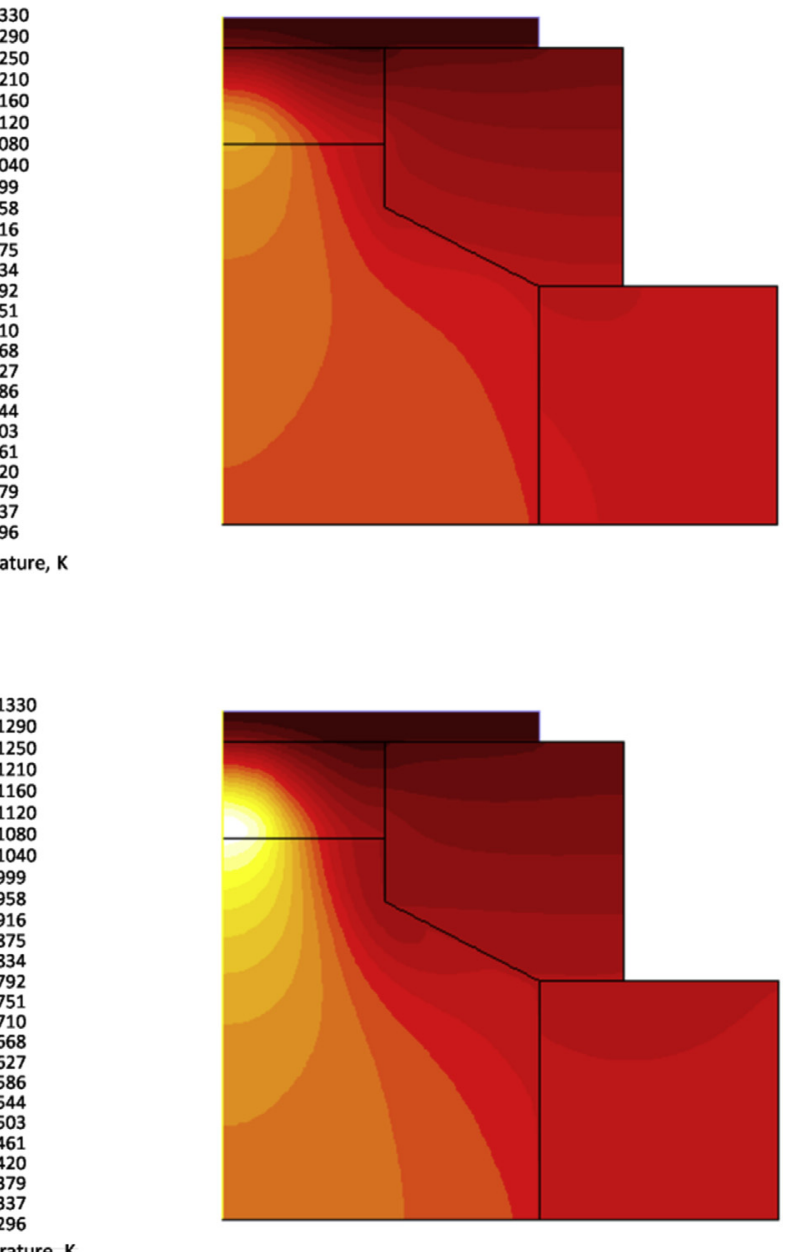

Fig. 11. Thermal distribution of gradual configuration in radial direction: a) increasing porosity; b) decreasing porosity.

depends on the temperature by the expression $\pm 0.004 \cdot|T|$, according to the standard IEC 584.2 (1982). This means that the measuring accuracy is $\pm 3.9 \mathrm{~K}$ at temperature ranges of the air at absorber outlet (964-981 K).

Simulation results deviate from the experimentally measured values by $1.7-2.8 \%(16.7-27.44 \mathrm{~K})$. This difference is due to the difficulty of taking high-temperature measurements in a fluid. In addition to the measuring error, the thermal radiation and the fluid flow influence the temperature values obtained. Furthermore, the numerical model is two dimensional, and the radiation heat transfer and the heat absorbed by the porous structure have been included in the volumetric-heat source. These conditions do not allow comparing experimental and numerical results using absolute values. For this reason, dimensionless evaluation has been regarded.

Moreover, other studies have considered acceptable deviations of $3.59 \%$ and $9.38 \%[48]$. This shows that the numerical results are in appreciable agreement with the experimental results, taking into account that deviations lower than $5 \%$ was obtained.

\subsection{Extinction-coefficient evaluation}

In order to determine the extinction coefficient for the two additional porosities considered ( 0.48 and 0.78 ), two simulations have been carried out, one for each porosity, with the same parameters considered for the experimental porosity. The deviation of
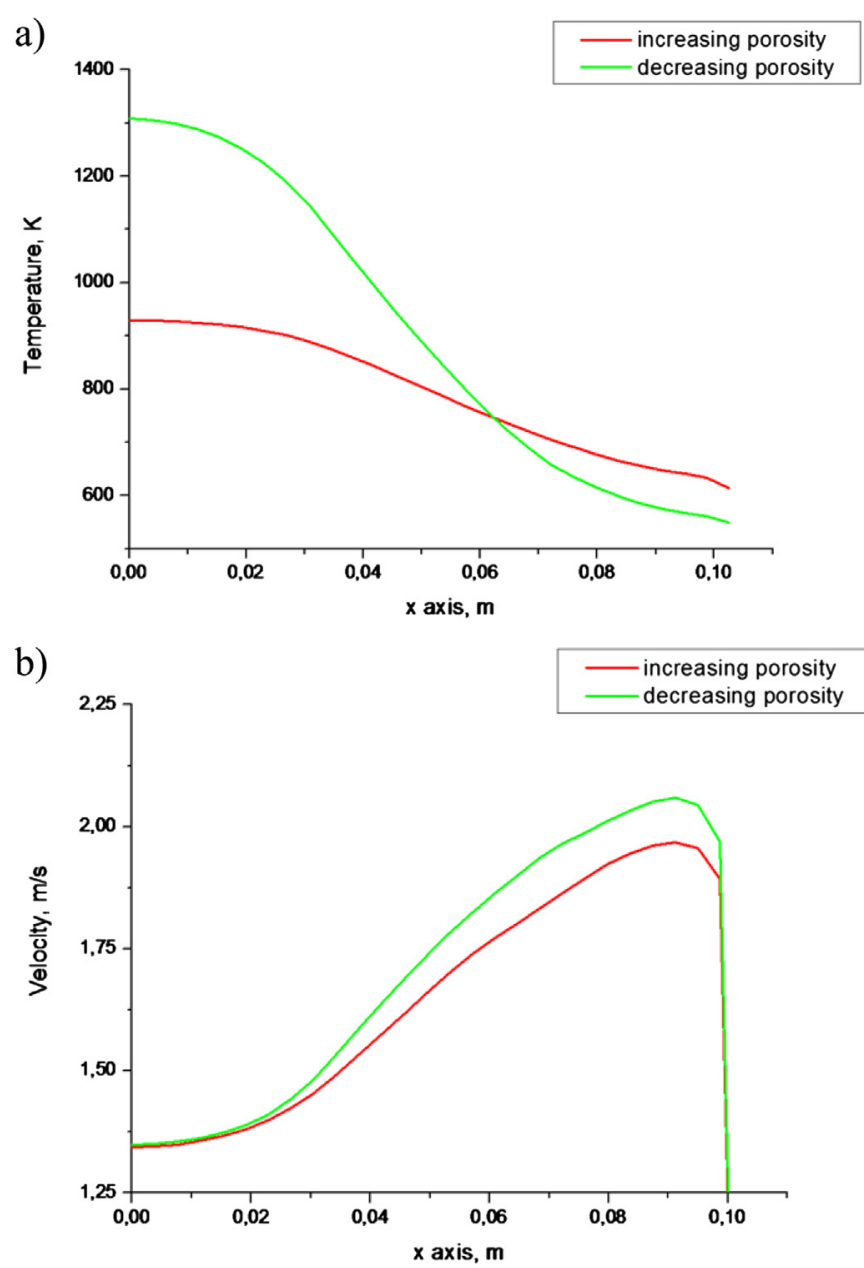

Fig. 12. Profiles at the porous-medium outlet for the gradual configuration in radial direction: a) thermal profile; b) velocity profile.

the heat-transfer coefficients has been used to evaluate the volumetric-heat source in each case, considering the reference value $\left(10^{07} \mathrm{~W} / \mathrm{m}^{3}\right)$, and a correction factor (see Table 3$)$. The extinction coefficients have been obtained analogously to the reference extinction coefficient in the Section 2.2.

Simulation results show that the extinction coefficient for a new porosity can be evaluated by means of a comparison between the heat-transfer coefficient of an experimental porosity, whose extinction coefficient is known, and the one obtained for the new porosity using the simulation conditions of the experimental case.

\subsection{Absorber material with constant porosity}

To optimize the porosity of the absorber, the simulation results of three different porosity values have been considered $(0.48,0.64$ and 0.78). The thermal distribution (Fig. 9) shows that the maximum temperature $(1058 \mathrm{~K})$ is reached by the highest porosity, because it allows a higher penetration of the incoming radiation into the absorber structure and presents a greater heat exchange surface.

Furthermore, the thermal and velocity profiles have been compared at the absorber outlet, and Fig. 10 shows that the highestporosity profiles present greater gradients than the others. This fact is going to have an influence in the material selection for the design of a volumetric absorber, because, in this case, it should have a higher resistance to thermal shock. Moreover, this effect is 
reinforced by the low air velocity at the centre, where an overheated area can be found due to the irradiance-peak location and a poor cooling in that region.

The average values of each profile have been compared with the experimental porosity (0.64), in order to evaluate the porosity influence on the air conditions at the absorber outlet. The results show that the porosity of 0.48 reaches average values, both temperature and velocity, around $7 \%$ lower and, the values for a porosity of 0.78 are around $4 \%$ higher.

In order to compare these results with those obtained from gradual configurations, new simulations of these constant porosities were carried out using an average value for the superficial heat source. This evaluation has been included in Tables 4 and 5 .

\subsection{Absorber material with gradual porosity in radial direction}

For the purpose of minimizing the overheating effect, by which the absorber material reaches a maximum temperature at the centre of the structure, two configurations with gradual porosity in radial direction were analyzed. One of them consists of an increasing porosity from a value of 0.48 at the centre of the porous medium up to an external porosity of 0.78 , and the other configuration considers a decreasing porosity.

a)
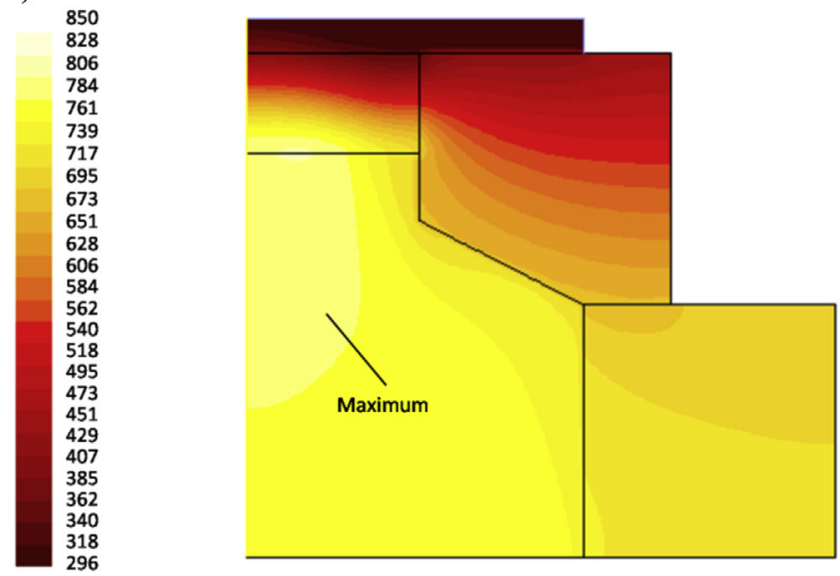

Temperature, $\mathrm{K}$

b)

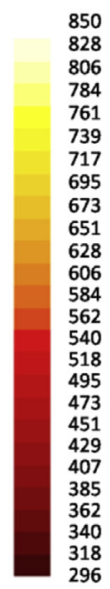

Temperature, $\mathrm{K}$

Fig. 13. Thermal distribution of gradual configuration according to depth: a) increasing porosity; b) decreasing porosity.

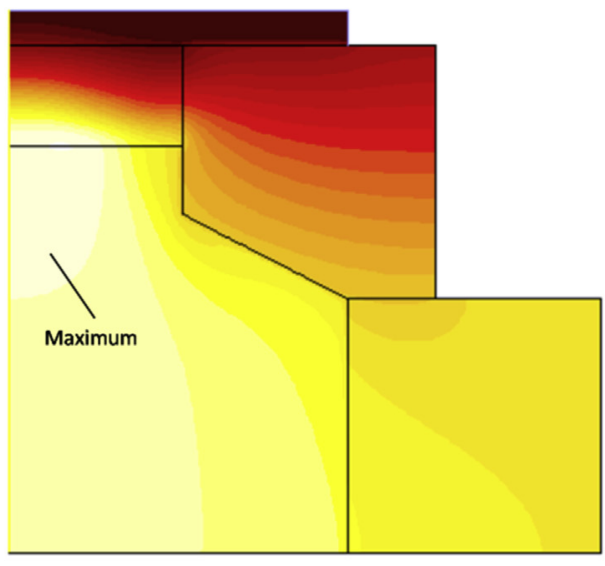

These configurations present a different thermal distribution in comparison to the previous cases (Fig. 11). The maximum temperature is concentrated in a smaller area at the centre of the absorber structure, and it corresponds to $938 \mathrm{~K}$ for increasing porosity and $1323 \mathrm{~K}$ in the case of decreasing porosity in radial direction.

The analysis of the temperature and velocity profiles shows that the decreasing configuration presents the greatest gradient for both variables (Fig. 12), being the velocity gradient lower than the thermal one. The temperature difference was around 58\%, considering the maximum and minimum values obtained from the simulation results at the absorber outlet of the decreasing configuration, and this shows the greatest overheating at the absorber centre in comparison with the constant-porosity configurations (Section 3.3). Therefore, the increasing-porosity configuration presents a more favourable behaviour due to the inherent limitations of the porous material. In this case, the increasing porosity has a lower thermal gradient than the decreasing one, thus allows preserving the absorber material from the thermal shock.

\subsection{Absorber material with gradual porosity according to depth}

Two different configurations have been regarded to analyze the effect of the gradual porosity according to depth. One considers an increasing porosity $(0.48-0.64-0.78)$ and the other $(0.78-0.64-$ 0.48 ) a decreasing one. In order to compare the gradual porosity in

a)

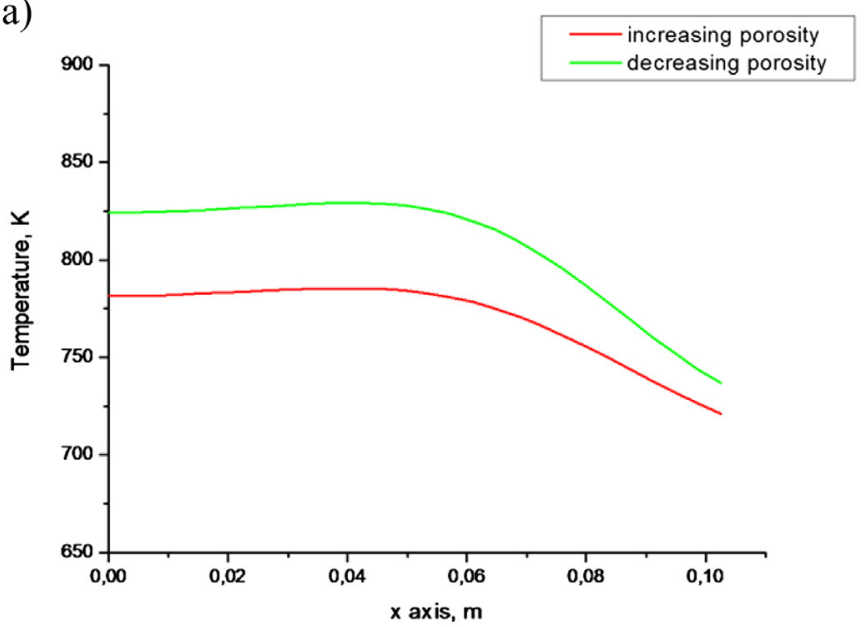

b)

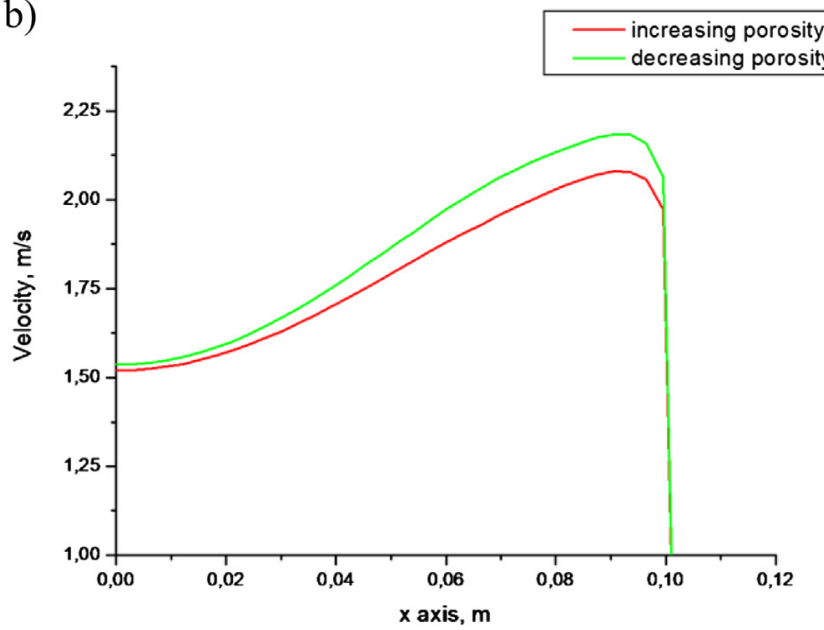

Fig. 14. Profiles at the porous-medium outlet for the gradual configuration according to depth: a) thermal profile; b) velocity profile. 
the radial direction with the one according to depth, an average value for the superficial heat source has been considered.

Simulation results (Fig. 13) show that the maximum temperature is reached by the decreasing porosity $(829 \mathrm{~K})$ located in a smaller area at the centre of the cavity (Fig. 13b) compared to the increasing one (Fig. 13a), which presents a lower temperature (maximum temperature of $785 \mathrm{~K}$ ). The difference between the two average-temperature values at the absorber outlet is around $4 \%$ and the velocity variation is $3.5 \%$ (Fig. 14), with a greater gradient for both variables in the decreasing configuration. In both cases, the velocity at the centre presents a minimum, because in the warmer area the permeability is lower and the air viscosity is higher. Both effects avoid the absorber cooling. This fact shows that this area is overheated, but this central velocity is greater than the one obtained for the radial configuration (1.5 and $1.3 \mathrm{~m} / \mathrm{s}$, respectively).

The results show that gradual porosity according to depth reaches a lower maximum temperature. However, the thermal distribution for this case is more homogeneous than for the gradual porosity in radial direction, because the radial variation avoids a homogeneous heat-flux distribution. It enhances the Gaussian effect of the irradiance peak at experimental conditions, strongly limiting the absorber durability in the overheated region.

\subsection{Thermal efficiency}

For the purpose of determining the best design for the solar volumetric receiver, the thermal efficiency has been evaluated by the following equation (Table 4):

$\eta=\frac{Q_{\text {conv }}}{Q_{\text {rec }}}$

where $\eta$ is the thermal efficiency, and $Q_{\text {conv }}$ is the convective flow, obtained from the energy balance between the fluid inlet and absorber outlet:

$Q_{\text {conv }}=m_{\mathrm{f}} \cdot c_{\mathrm{pf}} \cdot\left(T_{\mathrm{f}, \text { out }}-T_{\mathrm{f}, \text { in }}\right)$

where $m_{\mathrm{f}}$ is the air mass flow, $c_{\mathrm{pf}}$ is the average specific heat capacity of the air, $T_{\mathrm{f}, \text { in }}$ is the air temperature at the absorber inlet, and $T_{\mathrm{f} \text {,out }}$ is the air temperature at the absorber outlet.

$Q_{\text {rec }}$ is the heat absorbed from the incoming concentrated-solar radiation over the inlet receiver surface, whose evaluation considers the superficial heat source $\left(I_{\mathrm{SO}}\right)$ according to the frontal receiving area $(A)$.

$Q_{\text {rec }}=I_{\mathrm{S} 0} \cdot A$

In order to compare the constant-porosity cases with the gradual porosity, simulations with constant porosity have been developed considering an average value of $456,000 \mathrm{~W} / \mathrm{m}^{2}$ for the superficial heat source (equation (6)). In this case, the highest porosity achieves a thermal efficiency of $53.79 \%$ (see Table 4 ). It presents a thermal gradient of $96.3 \mathrm{~K}$ and its velocity variation corresponds to $0.72 \mathrm{~m} / \mathrm{s}$ at the absorber outlet (Table 5). Thus the configuration with a constant porosity of 0.78 increases the efficiency in around 6\% and the maximum temperature in around $71 \mathrm{~K}$ compared to the porosity of 0.48 .

In order to improve these results, a combination of three selected porosities was proposed. All gradual-porosity configurations present a higher thermal efficiency in comparison with the constant-porosity one, and the efficiency difference is between $12 \%$ and $28 \%$ with regard to the constant porosity of 0.78 .

The decreasing-porosity configurations achieve a greater thermal efficiency than those obtained by the increasing one (around 5-8\% higher), and gradual porosities according to depth reach the highest efficiencies (Table 4) with a lower thermal gradient than in the porosity radial variation (Table 5).

Therefore, the decreasing porosity according to depth is proposed as the best design, which achieves the highest thermal efficiency (81.99\%) with thermal and velocity gradients of $150.7 \mathrm{~K}$ and $0.65 \mathrm{~m} / \mathrm{s}$, respectively. These results show that the use of a greater porosity in the area which receives the solar radiation allows decreasing the heat losses to the environment, because the solar beams reach a greater penetration depth into the porous material.

\section{Conclusions}

Different configurations for a solar volumetric absorber have been analyzed by means of CFD software to study temperature and velocity distributions. Three constant porosities, two gradual porosities according to depth, and two gradual porosities in radial direction have been considered. The results obtained by this study can be considered as an initial prediction of the behaviour of gradual-porosity materials.

Various constant porosities and gradual configurations have been regarded in the analysis to develop new absorber designs. The thermal efficiencies reached by the proposed configurations are compared in order to define the best design.

Temperature and velocity show an increased distribution for higher porosities because of the greater heat absorbed. Consequently, the thermal efficiency increases, as shown in Table 4. The constant-porosity configuration presents the highest maximum temperature for the greatest porosity, and thermal efficiency is around $50 \%$.

The receiver with gradual porosities shows a significant increase of the thermal efficiency. On the one hand, the efficiency of gradual porosity according to depth is higher than the one obtained for the radial configuration. The greatest efficiency for the radial porosity variation corresponds to $72 \%$, and the one for the decreasingporosity variation according to depth is $82 \%$. The higher efficiency of this configuration is because it allows a greater solar-irradiance penetration into the absorber structure and a more homogeneous heat-flux distribution, minimizing the thermal gradient.

On the other hand, the gradual porosity in radial direction shows the overheating area located at the centre of the receiver with a greater temperature than the one obtained in the gradual porosity according to depth, but also presents the highest thermal gradient and a lower thermal efficiency due to the nonhomogeneous distribution of the heat flux. For this reason, this configuration is not appropriate for the design of a solar volumetric receiver.

However, fluid heating for the gradual porosity according to depth is more homogeneous, minimizing the thermal gradients in the absorber material. Therefore, the decreasing-porosity configuration according to depth has been proposed as an alternative to the constant-porosity design.

\section{Acknowledgements}

The authors would like to thank the European Commission for funding the project SFERA in the framework of which this study has been carried out.

Particularly, the first author wishes to thank Plataforma Solar de Almería and the University of Almería for the financial assistance/ scholarship awarded for the PhD research period during which this work was conducted. In addition, it is important to mention the help that the PSA Solar Furnace's staff has given to obtain the experimental results and special thanks go to Félix Téllez, Reiner Buck, Alfonso Vidal, José Rodríguez and Inmaculada Cañadas. 


\section{References}

[1] Hoffschmidt B, Téllez F, Valverde A, Fernández J, Fernández V. Performance evaluation of the 200-kW $\mathrm{kW}_{\mathrm{th}}$-HiTRec-II open volumetric air receiver. J Sol Energy Eng 2003;125:87-94.

[2] Gokona N, Osawaa Y, Nakazawaa D, Kodama T. Kinetics of $\mathrm{CO}_{2}$ reforming of methane by catalytically activated metallic foam absorber for solar receiverreactors. Int J Hydrogen Energy 2009;34:1787-800.

[3] Roeb M, Sattler C, Klüser R, Monnerie N, de Oliveira L, Konstandopoulos AG, et al. Solar hydrogen production by a two-step cycle based on mixed iron oxides. J Sol Energy Eng 2006;128:125-33.

[4] Villafán-Vidales HI, Abanades S, Caliot C, Romero-Paredes H. Heat transfer simulation in a thermochemical solar reactor based on a volumetric porous receiver. Appl Therm Eng 2011;31:3377-86.

[5] Chavez JM, Chaza C. Testing of a porous ceramic absorber for a volumetric air receiver. Sol Energy Mater 1991;24:172-81.

[6] Pitz-Paal R, Morhenne J, Fiebig M. A new concept of a selective solar receiver for high temperature applications. Sol Energy Mater 1991;24:293-306.

[7] Carotenuto A, Ruocco G, Reale F. Heat exchange in a multi-cavity volumetric solar receiver. Sol Energy 1991;46:241-8.

[8] Reale F, Ruocco G, Carotenuto A, Nocera U, Bonomo F. Final design of a multi cavity volumetric solar receiver. Sol Energy Mater 1991;24:284-92.

[9] Karni J, Kribus A, Rubin R, Doron P. The "porcupine": a novel high-flux absorber for volumetric solar receivers. J Sol Energy Eng 1998;120:85-95.

[10] Fend T, Hoffschmidt B, Pitz-Paal R, Reutter O, Rietbrock P. Porous materials as open volumetric solar receivers: experimental determination of thermophysical and heat transfer properties. Energy 2004;29:823-33.

[11] Fend T, Hoffschmidt B, Pitz-Paal R, Reutter O. Cellular ceramics use in solar radiation conversion. In: Scheffler $\mathrm{M}$, Colombo P, editors. Cellular ceramics: structure, manufacturing and applications. Weinheim: Willey-VCH GmbH \& Co. KgaA; 2005, p. 523-46.

[12] Becker M, Fend T, Hoffschmidt B, Pitz-Paal R, Reutter O, Stamatov V, et al. Theoretical and numerical investigation of flow stability in porous materials applied as volumetric solar receivers. Sol Energy 2006;80:1241-8.

[13] Marcos MJ, Romero M, Palero S. Analysis of air return alternatives for CRStype open volumetric receiver. Energy 2004;29:677-86.

[14] Wu Z, Caliot C, Bai F, Flamant G, Wang Z, Zhang J, et al. Experimental and numerical studies of the pressure drop in ceramic foams for volumetric solar receiver applications. Appl Energy 2010;87:504-13.

[15] Wu Z, Caliot C, Flamant G, Wang Z. Numerical simulation of convective heat transfer between air flow and ceramic foams to optimise volumetric solar air receiver performances. Int J Heat Mass Transfer 2011;54:1527-37.

[16] Xu C, Song Z, Chen L, Zhen Y. Numerical investigation on porous media heat transfer in a solar tower receiver. Renew Energy 2011;36:1138-44.

[17] Smirnova O, Fend T, Schwarzbözl P, Schöllgen D. Homogeneous and inhomogeneous model for flow and heat transfer in porous materials as high temperature solar air receivers. In: Proceedings of the COMSOL conference; 2010. Paris.

[18] Wua Z, Caliot C, Flamant G, Wang Z. Coupled radiation and flow modeling in ceramic foam volumetric solar air receivers. Sol Energy 2011;85:2374-85.

[19] Fend T, Pitz-Paal R, Reutter O, Bauer J, Hoffschmidt B. Two novel high-porosity materials as volumetric receivers for concentrated solar radiation. Sol Energy Mater Sol Cells 2004;84:291-304

[20] Veeraragavan A, Lenert A, Yilbas B, Al-Dini S, Wang EN. Analytical model for the design of volumetric solar flow receivers. Int J Heat Mass Transfer 2012;55:556-64.
[21] Lenert A, Wang EN. Optimization of nanofluid volumetric receivers for solar thermal energy conversion. Sol Energy 2012;86:253-65.

[22] Roldán MI, Cañadas I, Casas JL, Zarza E. Thermal analysis and design of a solar prototype for high-temperature processes. Int J Heat Mass Transfer 2013;56: 309-18.

[23] Roldán MI, Cañadas I, Casas JL. Simulation and thermal analysis of a solar receiver for high-temperature industrial process. In: SolarPACES conference; 2011. Granada, Spain.

[24] del Arco JA, Müller R, García JL. PSA solar furnace documentation. PSA's internal document; 1991.

[25] Ballestrín J, Monterreal R. Hybrid heat flux measurement system for sola central receiver evaluation. Energy 2004;29:915-24.

[26] Monterreal R. Datos del barrido del foco y del atenuador en el Horno Solar de la PSA, campaña de Marzo del 2008. PSA's internal document; 2008.

[27] Téllez F. Thermal performance evaluation of the $200 \mathrm{~kW}_{\mathrm{th}}$ "SolAir" volumetric solar receiver. Tech Rep CIEMAT; 2003. p. 1024.

[28] Holman JP. Transferencia de Calor. 8th ed. Madrid: McGraw-Hill; 1998.

[29] Palero S. Estudio teórico-experimental de la transferencia de calor en absorbedores solares volumétricos: estados críticos [Ph.D.]. CIEMAT-UNED; 2008.

[30] Blazek J. Computational fluid dynamics: principles and applications. 2nd ed. Elsevier; 2005.

[31] Batchelor GK. An introduction to fluid dynamics. Cambridge: University Press; 1967.

[32] Modeling heat transfer [Chapter 12], Fluent 6.2 user's guide. Lebanon, NH: Fluent-Inc.; 2005.

[33] Boundary conditions [Chapter 7], Fluent 6.2 user's guide. Lebanon, NH: Fluent-Inc.; 2005.

[34] Modelling turbulence [Chapter 11], Fluent 6.2 user's guide. Lebanon, NH Fluent-Inc.; 2005.

[35] Launder BE, Spalding DB. The numerical computation of turbulent flows. Comput Methods Appl Mech Eng 1974;3:269-89.

[36] The Gambit graphical user interface [Chapter 3], Gambit 2.2 user's guide. Lebanon, NH: Fluent-Inc.; 2004.

[37] Modeling basic fluid flow [Chapter 9], Fluent 6.2 user's guide. Lebanon, NH: Fluent-Inc.; 2005.

[38] Becerra Santiago LO, Guardado González ME. Estimación de la incertidumbre en la determinación de la densidad del aire. Centro Nacional de Metrología de México; 2001.

[39] Crane Company. Flow of fluids through valves, fittings, and pipe. Technica paper; 1988. p. 410.

[40] Chemical Rubber Company (CRC). CRC handbook of chemistry and physics. 65th ed. CRC Press; 1984.

[41] Physical properties [Chapter 8], Fluent 6.2 user's guide. Lebanon, NH: FluentInc.; 2005.

[42] Barrú Hayas A. Thermodynamics of moist air. Spain: Department of Physics, University of Jaén; 2007.

43] Belmonte Calera A, Barbero AJ. Atmospheric thermodynamics. Spain Department of Applied Physics, University of Castilla-La Mancha; 2008.

[44] Wolfram Alpha Computational Knowledge Engine. Thermal conductivity of moist air. Wolfram Research Company; 2009.

45] ThermalCeramics. Data sheet of dense concretes Firecrete ${ }^{\mathrm{TM}} 2001$.

[46] Dantzig JA, Tucker III CL. Modelling in materials processing. 1st ed. Cambridge University Press; 2001.

[47] Using the solver [Chapter 26], Fluent 6.2 user's guide. Lebanon, NH: FluentInc.; 2005.

[48] Ozalp N, JayaKrishna D. CFD analysis on the influence of helical carving in a vortex flow solar reactor. Int J Hydrogen Energy 2010;35:6248-60. 